\title{
Multistatic SAR Satellite Formations: Potentials and Challenges
}

\author{
Gerhard Krieger, Alberto Moreira \\ Microwaves and Radar Institute, German Aerospace Centre (DLR) \\ 82234 Oberpfaffenhofen, Germany, E-Mail: Gerhard.Krieger@dlr.de
}

\begin{abstract}
This paper discusses the capabilities of multistatic SAR satellite configurations for different applications like high resolution DEM generation using multibaseline single-pass crosstrack interferometry, 3-D vegetation mapping and layover solution with spaceborne SAR tomography, high resolution wide swath SAR imaging with sparse satellite arrays, multibaseline velocity estimation of moving objects and scatterer fields, spatial and radiometric resolution enhancement in SAR images, and multistatic imaging for improved detection and classification. Furthermore, some major challenges like phase and time synchronisation, multistatic SAR processing, satellite orbit selection, and relative position sensing will be addressed.
\end{abstract}

\section{INTRODUCTION}

Multistatic synthetic aperture radar operates with multiple receive antennas which are distributed among different platforms. Such a spatial separation has several operational advantages which will increase the capability, reliability and flexibility of future SAR missions [1]. Potential application areas of multistatic SAR systems include single-pass cross-track and along-track interferometry, spaceborne tomography, wide swath imaging, resolution enhancement, interference suppression, ground moving target indication, and multistatic SAR imaging. The simultaneous data acquisition with multiple satellites avoids temporal and atmospheric disturbances, improves the performance, and enables the detection of fast changes.

Multistatic SAR systems may be divided into semi-active and fully-active configurations. Semi-active configurations combine an active illuminator with one or more passive receivers as shown in Fig. 1 on the left. This allows for the use of low-cost receive-only microsatellites, thereby enabling a cost-efficient implementation of single-pass SAR systems with multiple baselines. Examples for semi-active configurations are the Interferometric Cartwheel [2] and Bissat [3]. In a fully active configuration, each radar has both transmit and receive capabilities as illustrated in Figure 1 on the right. Fully active systems can be operated in many different modes, thereby providing increased flexibility, easy phase synchronization, and higher redundancy. Examples for fully-active systems are TechSAT 21 [4] and TanDEM-X [5]. The following sections discuss the potentials and challenges of such systems.
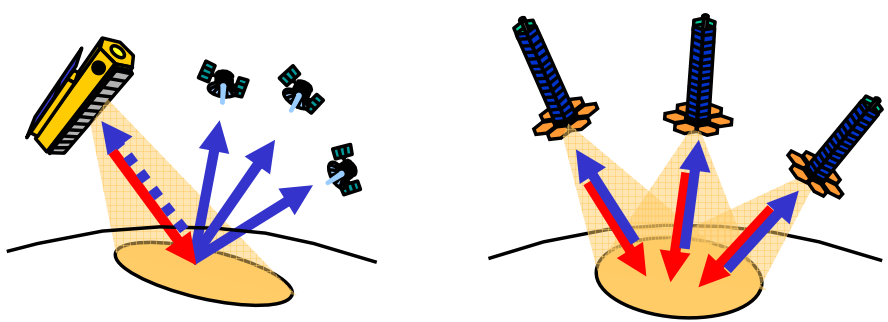

Figure 1. Semi-active (left) and fully-active (right) multistatic SAR systems.

\section{Multi-BASEline CROSS-TRACK INTERFEROMETRY}

The increased bandwidth of future spaceborne SAR systems enables coherent interferometric data acquisitions with large cross-track baselines. Such baselines are well suited to reduce the impact of system induced errors due to limited SNR or limited baseline knowledge. However, large baselines are also associated with an increased susceptibility to height ambiguities in case of DEM generation. The classical technique to resolve such ambiguities is phase unwrapping, which exploits the correlated structure of natural topography caused by gravitational forces and erosion. While it may be expected that such techniques profit from the improved spatial resolution of future SAR sensors, it is also clear that classical phase unwrapping runs into its limits in case of large baseline acquisitions of e.g. rough topography [6]. For example, the currently planned TanDEM-X mission will make use of only ca. $2 \%$ of the critical baseline for operational DEM generation [7], thereby neglecting the great potential to increase the height accuracy by one order of magnitude down to the decimeter or even centimeter level.

Recently, several innovative techniques have been suggested to resolve phase ambiguities in large baseline interferograms. One approach is based on the determination of the absolute phase by estimating the mutual range shift between corresponding pixels of two complex SAR images [8][9]. The left hand side of Fig. 2 shows predictions of the resulting height accuracy for TerraSAR-L, TerraSAR-X, and ERS/Envisat. In this example, we assumed a perpendicular baseline corresponding to $10 \%$ of the critical baseline, a coherence of 0.8 which is an optimistic value for a repeat pass scenario but a good approximation for e.g. a TerraSAR-L cartwheel-like configuration [10], and an independent post-spacing of $10 \mathrm{~m}$ for TerraSAR-L and TerraSAR-X (30m for Envisat/ERS).
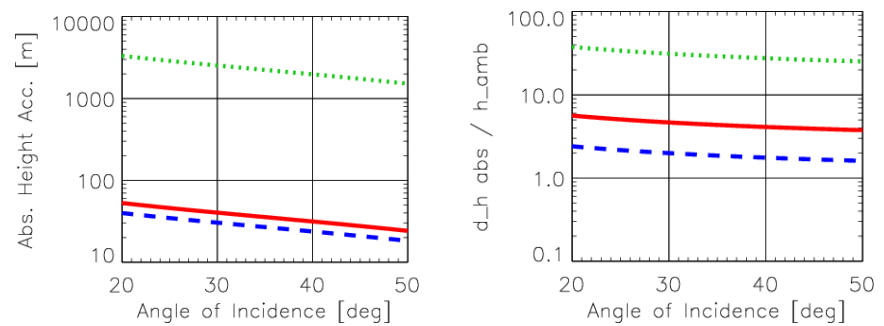

Figure 2. Height accuracy predicted from Cramer-Rao bound for differential shift esimtation (cf. [9]) for $10 \%$ of the critical baseline (left) and ratio

between absolute height accuracy and height of ambiguity (right). Solid: TerraSAR-X (150MHz, 10m posting). Dashed: TerraSAR-L (85MHz, 10m posting). Dotted: ERS1/2 viz. ASAR/Envisat (16MHz, 30m posting).

The right hand side of Fig. 2 compares the absolute height accuracy with the corresponding height of ambiguity resulting from an interferometric combination of the SAR images. It becomes clear, that the resulting accuracy is not sufficient to 
resolve the interferometric phase ambiguities which would require a ratio $\Delta \mathrm{h}_{\mathrm{abs}} / \mathrm{h}_{\mathrm{amb}}<<1$.

Several other techniques have been suggested to resolve phase ambiguities by a combination of multiple SAR images acquired with different baselines [11]-[15]. Some of these techniques evaluate the phase increments between adjacent pixels in multiple co-registered interferograms, thereby taking advantage of the deterministic relation between the baseline length and the phase gradient [11]. Other techniques resolve height ambiguities independently for each pixel [12], e.g. in an Earth based coordinate system by using a maximum likelihood approach [13]. This offers the opportunity to fuse multiple height estimates acquired in completely different imaging geometries, but requires also a precise knowledge of the remainder phase (more precisely: remainder topographic height) for each interferometric image pair. Still other techniques regard height determination as a direction of arrival (DOA) estimation in a sparse array and apply e.g. model based spectral estimation techniques to fuse the information from multiple SAR images in an unambiguous height estimate [14]. Interferometric acquisitions with large baselines may also use a joint multichannel processing to minimize deteriorations from slope dependent baseline decorrelation [15].

These multibaseline techniques can also be combined with the differential ranging approach mentioned before. By this, it becomes possible to bridge the 'performance' gap between the height accuracy achievable from differential ranging and the height of ambiguity associated with interferometric signal evaluation. This is illustrated in Fig. 3 for a semi-active TerraSAR-L formation which provides multiple cross-track baselines at a selectable baseline ratio in one single pass [10]. The performance example on the right shows that it is possible to increase the height of ambiguity by a factor of 10 if the signals from two interferometric baselines are combined. A combination of these two interferograms with the absolute phase from differential ranging is hence sufficient to recover the absolute phase in the large baseline interferogram. Using an even larger (additional) baseline could then push the performance up to the limits imposed by the critical baseline. The multistatic SAR in Fig. 3 acquires all interferometric data in a single pass using the same imaging geometry, thereby enabling an efficient fusion of the SAR image stack in radar slant range geometry. Further opportunities arise from a joint evaluation of multibaseline coherence, which reflects important characteristica of both volume and surface scatterers.

Multibaseline data acquisitions are also of great advantage for Polarimetric SAR Interferometry (PolInSAR) [16][17]. For example, the analyses in [18] demonstrate the intricate connection of the PolInSAR performance with radar instrument settings, baseline length, and volume height. The availability of multiple baselines would hence allow a space variant selection of those baselines which provide an optimum separation between the vertical phase centers, thereby improving the PolInSAR performance and avoiding phase ambiguities. Further improvements are expected by a joint evaluation of all multibaseline SAR signals [19][20], but optimized techniques have still to be developed for the great variety of potential scatterer and application scenarios. PolInSAR is furthermore well suited for DEM generation since it enables a distinction between digital terrain models (DTM) and digital surface models (DSM) which are both of high interest for a broad spectrum of scientific and commercial applications.
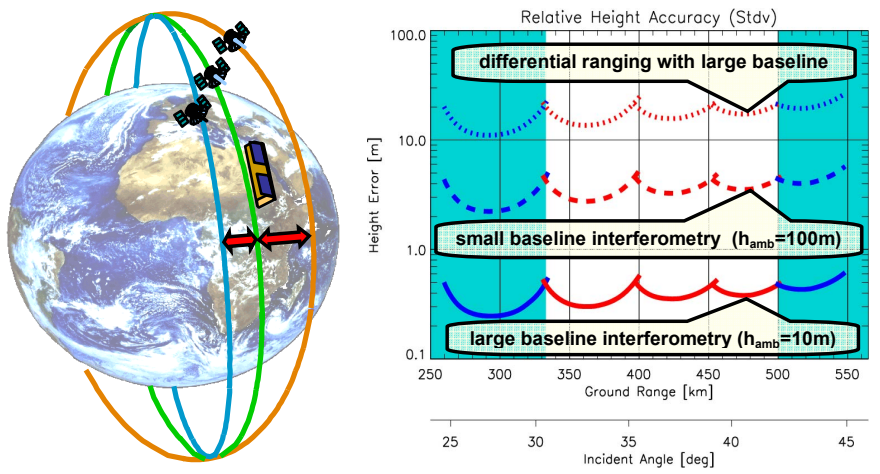

Figure 3. Multibaseline single-pass data acquisition in Pendulum configuration (left) and predicted DEM performance (right). The interferometric height accuracy is shown for two baselines corresponding to a height of ambiguity of 100 $\mathrm{m}$ (dashed) and $10 \mathrm{~m}$ (solid). The dotted line is the predicted height accuracy for differential ranging.

\section{SAR TOMOGRAPHY}

A constellation of multiple radar satellites recording the scattered signals from a common illuminated footprint can also be regarded as a large aperture system with sparsely distributed subaperture elements. The combination of multiple receiver signals can hence be treated in the framework of array processing. A prominent example is SAR tomography, which combines the signals from several receivers to form a sparse aperture perpendicular to the flight direction [21]-[25]. This enables a real 3-D imaging of semitransparent volume scatterers like e.g. vegetation, dry soil, sand, or ice. SAR tomography has furthermore the potential to resolve SAR image distortions due to layover and foreshortening [23][25]. Besides some laboratory experiments, SAR tomography has up to now only been demonstrated by using multiple passes either in an airborne [22] or a spaceborne configuration [24]. In these scenarios, major problems arise from temporal decorrelation and unevenly spaced passes, which both limit the achievable performance and require a sophisticated processing to avoid high sidelobes in the tomographic response. Pendulum-like satellite formations as shown in Fig. 3 are well suited to overcome such limitations by providing multiple baselines at a precisely adjustable baseline ratio in a single pass. Further advantages of single-pass multistatic data acquisitions are significantly improved baseline knowledge, no distortions due to systematic scatterer movements, the cancellation of atmospheric disturbances, and better predictability of the noise level within each channel, thereby improving the inversion performance significantly. The minimization of such disturbances becomes even more important, as advanced SAR tomography techniques try to incorporate more and more a priori information in the multibaseline inversion process to e.g. increase the resolution beyond the Rayleigh limit given by the maximum baseline length (an extreme example is conventional DEM generation, which assumes exactly one vertical layer within each resolution cell).

Tomographic techniques can also be combined with applications from cross-track interferometry. One example is layover resolution in conjunction with multibaseline DEM generation. This enables data takes with steep incident angles, thereby increasing the SNR and avoiding DEM voids due to 
shadows. Steep incident angle imaging is also of high interest for urban environments and deep valleys. First steps in this direction have already been made in [25], but further work is required to apply such techniques to a real world environment with e.g. multipass effects in urban areas, uneven satellite orbit spacing, unknown number of layover layers, etc. Additional baselines for layover solution can also be acquired with multiple passes, but the spatiotemporal processing should then clearly differentiate between the errors from the highly coherent single pass acquisitions and the less coherent repeat pass acquisitions to optimize the performance.

\section{SUPPRESSION OF RANGE AND AZIMUTH AMBIGUITIES AND RESOLUTION ENHANCEMENT}

Another opportunity of a sparse satellite array is the formation of very narrow antenna beams to suppress range and azimuth ambiguities during the SAR image generation [26][30]. This will in turn lead to a reduction of the required antenna size for each receiver, thereby enabling cost-effective and powerful SAR missions with broad coverage and high resolution. The opportunity for high resolution wide swath SAR imaging avoids conflicts from operating SAR systems in mutually exclusive imaging modes like ScanSAR, Stripmap, and Spotlight. This enables regular observations of large areas, satisfies a wider user community, and facilitates mission planning. A further potential is efficient interference suppression, which will become more and more important as the congestion of the frequency spectrum by an ever rising number of different users increases continuously. Sparse array beamforming could also be combined with digital beamforming within each receiver (cf. Fig. 4). This combination of small and large array beamforming is well suited to improve the performance [1][27].

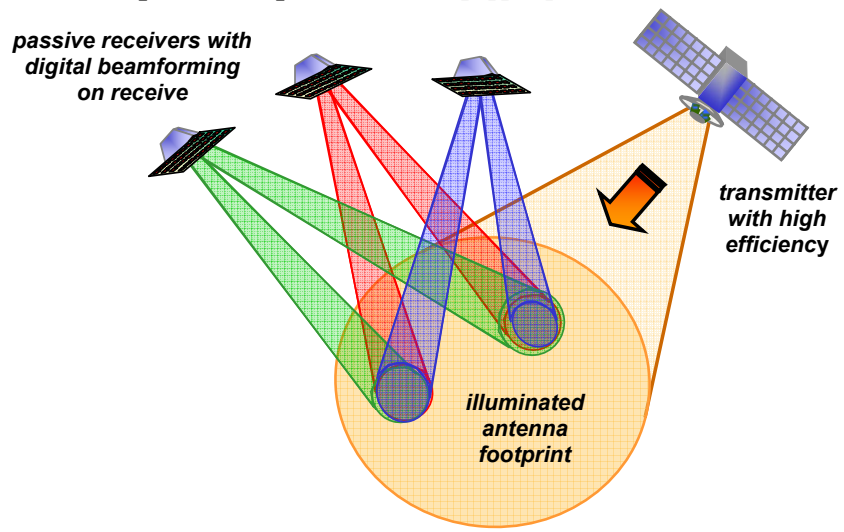

Figure 4. Sparse aperture SAR with digital beamforming on receive.

A coherent combination of multiple SAR images acquired from different incident angles can also enhance the geometric resolution [31][2]. This super-resolution technique may again be regarded as the formation of narrow beams which divide each resolution cell into multiple sub-cells. Multiple SAR images can furthermore be combined incoherently to reduce speckle and to improve the radiometric resolution without any loss of spatial resolution. Such techniques are of high interest to alleviate the bandwidth limitations for spaceborne SAR sensors posed by international frequency regulations.

Range and azimuth ambiguity suppression can also be combined with multibaseline SAR interferometry or tomography [1]. For this, it becomes necessary to consider the complete 3-D signal focusing from raw data, and not, as usually done in SAR interferometry and tomography, only the 1-D operator applied to the stack of range and azimuth focused SAR images.

\section{Multistatic SAR IMAGING}

Multistatic SAR can simultaneously provide multiple SAR images with different viewing geometries, thereby increasing the observation space considerably. This facilitates the detection, classification, and recognition of both natural and artificial objects [32][33]. Multistatic observations are furthermore of great interest for measurements of surface and vegetation parameters [34][35]. The ONERA-DLR bistatic SAR experiment revealed that even small bistatic angles may already cause a notable change of the scattering behavior [36]. A joint evaluation of mono- and bistatic SAR images could also be used to isolate different scattering mechanisms, like e.g. a distinction between highly directive dihedral returns from more isotropic volume scattering. Such bi- and multistatic scattering profiles could further be augmented with interferometric information. The evaluation of multistatic SAR images can furthermore use indirect information, e.g. systematic variations of the shadow pairs seen by each receiver [37]. Additional potentials arise for and from radargrammetry [38]. Multistatic SAR imaging enables also a precise localization and tracking of small objects in 3-D space [39].

High resolution bi- and multistatic SAR data can also be acquired with nadir-looking receivers [1]. This enables a simultaneous data acquisition of the same area with other remote sensing instruments like altimeters, lidars, or optical/hyperspectral sensors. The data from the different sensors may then be combined for such different purposes as DEM calibration, orthorectification, or multi sensor object and scene classification.

\section{ALONG-TRACK INTERFEROMETRY AND MOVING OBJECT INDICATION}

Multistatic SAR satellite formations are predestinated for large baseline along-track interferometry (ATI), which compares the phase of two or more complex SAR images acquired in identical geometries but separated by short time intervals [40]-[45]. This technique is hence well suited for the monitoring of dynamic processes. A prominent ATI application is the measurement of ocean and tidal currents. Large alongtrack baselines are required for accurate measurements of slow movements, while short baselines are required to avoid ambiguities in case of higher velocities. Hence, an acquisition with multiple along-track baselines would be of great help to resolve ambiguities, thereby enabling improved and more accurate measurements over a wide spectrum of potential scatterer velocities [44][45]. Multistatic formations with three or more receivers allow furthermore for the reliable detection, localization, and velocity estimation of even slowly moving objects on the ground [46]-[49]. Due to the large separation between the individual receiver satellites, efficient clutter suppression may be achieved, thereby enabling the detection and localization of weak scatterers with low signal to clutter ratio. The large receiver separation allows also for highly accurate velocity estimates and mitigates the problem of blindness against certain directions of scatterer movement. This is e.g. of high importance for future spaceborne traffic monitoring systems [50]. 


\section{REPEAT-PASS SPARSE ARRAY SAR}

Further opportunities arise from a comparison of several multistatic data sets acquired during different passes of the satellite formation. For example, the combination of single pass and repeat pass interferograms will have great potential for such different applications as the detection of the grounding line which separates the shelf from the inland ice, monitoring of vegetation growth, mapping of atmospheric water vapor with high spatial resolution, measurement of snow accumulation or detection of anthropogenic changes of the environment, e.g. due to deforestation. Note that most of these combinations rely on a comparison of two or more single-pass cross-track interferograms and do hence not necessarily require coherence between the different passes. Further information may be gained from an evaluation of coherence changes between different passes, potentially augmented by polarimetric information. This may e.g. reveal even slight changes in the soil and vegetation structure.

The information space of a multi-pass sparse aperture SAR may further be increased by varying some system parameters between different passes. One example is a PolInSAR system which acquires the interferometric data in a single pass and the different polarizations in subsequent passes. This ensures high coherence within the interferometric channels, while simplifying the mission design [18]. Another example is a systematic change of the viewing geometry between different passes. This is e.g. well suited to resolve errors during DEM generation in mountainous terrain where steep slopes cause foreshortening, layover, and shadows [6]. SAR interferograms from different passes can furthermore be used to calibrate interferometric data acquisitions, e.g. by block adjustment from crossing orbits.

Bi- and multistatic data acquisitions are also well suited to separate the topographic (and in some cases also the atmospheric) phase from scatterer movements between different passes, thereby facilitating highly accurate measurements of even small terrain and object displacements [51]-[53]. Further potentials arise from differential tomography [54].

\section{DISCUSSION}

The previous sections revealed the great potential of multistatic SAR satellite formations for a wealth of powerful remote sensing applications. Most of these applications require close satellite formations. Hence, orbit selection and collision avoidance may become a major design driver. For example, the Pendulum formation in Fig. 3 requires a sufficient along-track separation between the satellites to exclude any collision risk at the northern and southern turns. This may be achieved by autonomous control [43]. An alternative is a slight modification of the satellite formation such that the orbits have a small vertical separation at the intersection of the orbital planes. This results in a HELIX like movement without orbit crossing [55][56]. The satellites may now be shifted arbitrarily along their individual orbits. This enables almost vanishing alongtrack baselines for a given latitude range. Very short along-track baselines are e.g. desired in case of DEM generation to avoid residual temporal decorrelation for some types of vegetation, or for interferometric data acquisitions of the ocean surface. The opportunity for safe satellite operation using the HELIX concept allows also for a closer distance between the transmitter and the passive receivers in cartwheel-like satellite formations, thereby enabling a potential increase of the coherent data space with monostatic SAR images from the transmitter.
Close satellite formations enable also a highly accurate measurement of the relative satellite positions by evaluating GPS differential carrier phase signals. This improves the baseline knowledge by at least one order of magnitude if compared with the difference of independently estimated orbit state vectors used in conventional repeat pass interferometry. Since formation flying satellites are also exposed to highly correlated orbit perturbations, it becomes possible to further reduce the impact of residual baseline estimation errors [7]. Close formations allow also for a precise adjustment of the inter-satellite baselines as required by several interferometric and tomographic applications. Such a baseline adjustment is orders of magnitude less sensitive to slight changes of gravitational forces than the fuel consuming baseline control in repeat orbits.

A further challenge is phase and time synchronization. Oscillator stability is of special concern in bi- and multistatic SAR systems, since there is no cancellation of low frequency phase errors as in a monostatic SAR where the same oscillator signal is used for modulation and demodulation. As discussed in [1][57], phase errors may cause a time variant shift, spurious sidelobes and a broadening of the bistatic SAR impulse response, as well as phase errors in the focused signal. Hence, appropriate calibration strategies are required to avoid such errors, especially in case of interferometric data acquisitions. One solution is a bidirectional phase synchronization link between each receiver pair, e.g., by radiating the USO signal [58] or by transmitting dedicated RF pulses as implemented in the TanDEM-X mission [5]. The use of higher synchronization frequencies has the advantage to minimize potential errors from the ionosphere [59]. Fully active systems may also evaluate the scattered signals from the common illuminated footprint, either by switching the transmitter from pulse to pulse [60], or by a simultaneous transmission of orthogonal waveforms (e.g. different RF subbands), thereby avoiding the necessity of dedicated antennas for phase referencing. An alternative is the use of oscillators with significantly improved long-term stability [61] in combination with a sparse net of calibration targets. Phase errors in multistatic SAR could in principle also be reduced by comparing the signals from multiple baselines, analyzing multiple azimuth looks, comparing the azimuth shift between monostatic and bistatic SAR images, and evaluating the signals from multiple passes. Absolute DEM calibration could furthermore take advantage from large baseline stereogrammetric radar data acquired in repeat passes (e.g. by evaluating monostatic data with large incident angle differences). Such data are then well suited to eliminate low frequency height errors caused by both insufficient oscillator synchronisation and limited baseline estimation accuracy.

Further challenges arise from bi- and multistatic SAR processing [1]. The previous sections exemplified a wealth of different remote sensing applications, each relying on a different processing of the information in the single-pass 3-D (or multipass 4-D) data cube acquired with a multistatic SAR. In the future, many of these applications can be combined, like e.g. high resolution wide swath imaging with SAR interferometry or tomography, thereby mutually improving the performance. Such combinations enable an extremely versatile SAR satellite formation with compact antennas serving a broad spectrum of powerful remote sensing applications. 


\section{REFERENCES}

[1] G. Krieger, A. Moreira, Spaceborne Bi- and Multistatic SAR: Potentials and Challenges, to appear in IEE Proc. Radar Sonar Navigation, 2005.

[2] D. Massonnet, Capabilities and Limitations of the Interferometric Cartwheel, IEEE Trans. Geosci. Remote Sensing 39 (3), pp. 506-520, 2001.

[3] A. Moccia, G. Rufino, M. D'Errico, G. Albertini, G. Salzillo, BISSAT: A Bistatic SAR for Earth Observation, IGARSS 2002, Toulouse, France.

[4] M. Martin, P. Klupar, S. Kilberg, J. Winter, Techsat 21 and Revolutionizing Space Missions using Microsatellites, 15th American Institute of Aeronautics and Astronautics Conference on Small Satellites 2001, Utah, USA.

[5] A. Moreira, G. Krieger, I. Hajnsek, M. Werner, D. Hounam, S. Riegger, E. Settelmeyer, TanDEM-X: A TerraSAR-X Add-On Satellite for Single-Pass SAR Interferometry, IGARSS 2004, Anchorage, USA.

[6] M. Eineder, Problems and Solutions for InSAR Digital Elevation Model Generation in Mountainous Terrain, Proc. Fringe 2003 Workshop, Frascati, Italy.

[7] G. Krieger, H. Fiedler, I. Hajnsek, M. Eineder, M. Werner, A. Moreira, TanDEMX: Mission Concept and Performance Analysis, IGARSS 2005, Seoul, Korea.

[8] S.N. Madsen, On Absolute Phase Determination Techniques in SAR Interferometry, SPIE Conf. Radar Sensor Techn. Vol. 2478, pp. 393-401, 1995.

[9] R. Bamler, M. Eineder, Accuracy of Differential Shift Estimation by Correlation and Split-Bandwidth Interferometry for Wideband and Delta-k SAR Systems, IEEE Geosci. Remote Sens. Letters, Vol. 2, No. 2, pp. 151-155, 2005.

[10] M. Zink, G. Krieger, T. Amiot, Interferometric Performance of a Cartwheel Constellation for TerraSAR-L, FRINGE Workshop 2003, Frascati, Italy.

[11] A. Ferretti, A. Monti Guarnieri, C. Prati, F. Rocca, Multi Baseline Interferometric Techniques and Applications, in ESA Workshop on Applications of ERS SAR Interferometry 1996, Zurich, Switzerland.

[12] D. Massonnet, H. Vadon, M. Rossi, Reduction of the Need for Phase Unwrapping in Radar Interferometry, IEEE Trans. Geosci. Remote Sens., Vol. 32, No. 2, pp.489-497, 1996

[13] M. Eineder, N. Adam, A Maximum-Likelihood Estimator to Simultaneously Unwrap, Geocode, and Fuse SAR Interferograms from Different Viewing Geometries into one Digital Elevation Model, IEEE Trans. Geosci. Remote Sens., Vol. 43, No. 1, pp. 24-36, 2005.

[14] F. Lombardini, H. Griffiths, Optimum and Suboptimum Estimator Performance for Multibaseline InSAR, Frequenz, Vol. 55, pp. 114-118, 2001.

[15] G. Fornaro, A. Monti Guarnierei, A. Pauciullo, F. Rocca, Joint Multibaseline SAR Interferometry, to appear in EURASIP Journal on Applied Signal Processing, 2005.

[16] S. Cloude, K. Papathanassiou, Polarimetric SAR Interferometry, IEEE Trans. Geosci. Remote Sensing, Vol. 36, No. 5, 1998

[17] K. Papathanassiou and S. Cloude, Single Baseline Polarimetric SAR Interferometry, IEEE Trans. Geosci. Remote Sens., Vol, 39, No. 11, pp. 23522363, 2001.

[18] G. Krieger, K. Papathanassiou, S. Cloude, Spaceborne Polarimatric SAR Interferometry: Performance Analysis and Mission Concepts, to appear in EURASIP Journal on Applied Signal Processing, 2005.

[19] R. Treuhaft, B. Law, G. Asner, S. Hensley, Vegetation Profile Estimates from Multialtitude, Multifrequency Radar Interferometric and Polarimetric Data, IGARSS 2000, Honolulu, USA.

[20] S. Cloude, M. Williams, A Coherent EM Scattering Model for Dual Baseline PollnSAR, IGARSS 2003, Toulouse, France.

[21] J. Homer, I. D Longstaff, and G. Callaghan, High Resolution 3-D SAR via MultiBaseline Interferometry, IGARSS 1996.

[22] A. Reigber and A. Moreira, First Demonstration of Airborne SAR Tomography using Multibaseline L-Band Data, IEEE Trans. Geosci. Remote Sensing, Vol. 38, No. 5, pp. 2142-2152, 2000.

[23] F. Lombardini, M. Montanari, F. Gini, Reflectivity Estimation for Multibaseline Interferometric Radar Imaging of Layover Extended Sources, IEEE Trans. Signal Processing, Vol. 51, No. 6, pp. 1508-1519, 2003

[24] G. Fornaro, F. Lombardini, F. Serafino, Three-Dimensional Multipass SAR Focusing: Experiments with Long-Term Spaceborne Data, IEEE Trans. Geosci. Remote Sens., Vol. 43, No. 4, pp. 702-714, 2005.

[25] F. Gini, F. Lombardini, M. Montanari, Layover Solution in Multibaseline SAR Interferometry, IEEE Trans. Aerospace Electr. Systems, Vol. 38, No. 4, pp. 1344$1356,2002$.

[26] N.A. Goodman, S.C. Lin, D. Rajakrishna, J.M. Stiles, Processing of MultipleReceiver Spaceborne Arrays for Wide Area SAR, IEEE Trans. Geosci. Remote Sensing, Vol. 40, No. 4, pp. 841-852, 2002.

[27] G. Krieger, A. Moreira, Potentials of Digital Beamforming in Bi-and Multistatic SAR, IGARSS 2003, Toulouse, France.

[28] J.P. Aguttes, The SAR Train Concept: Required Antenna Area Distributed over N Smaller Satellites, Increase of Performance by N, IGARSS 2003, Toulouse, France.

[29] M.. Younis, Digital Beam-Forming for High-Resolution Wide Swath Real and Synthetic Aperture Radar, PhD-thesis, Karlsruhe, Germany, 2004.
[30] G. Krieger, N. Gebert, A. Moreira, Unambiguous SAR Signal Reconstruction From Nonuniform Displaced Phase Center Sampling, IEEE Geoscience and Remote Sensing Letters, Vol. 1, No. 4, 2004.

[31] C. Prati and F. Rocca, Improving Slant-Range Resolution with Multiple SAR Surveys, IEEE Trans. Aerospace Electronic Systems, Vol. 29, pp. 135-143, 1993.

[32] J. Glaser, Some Results in the Bistatic Radar Cross Section (RCS) of Complex Objects, Proc. IEEE, Vol. 77, pp. 639-648, 1989.

[33] R. Eigel, P. Collins, A. Terzuoli, G. Nesti, J. Fortuny, Bistatic Scattering Characterization of Complex Objects, IEEE Trans. Geosci. Remote Sensing, Vol. 38, No. 5, pp. 2078-2092, 2000.

[34] F. Ulaby, T. van Deventer, J. East, T. Haddock, M.E: Coluzzi, Millimeter-Wave Bistatic Scattering from Ground and Vegetation Targets, IEEE Trans. Geosci. Remote Sensing, Vol. 26, No. 3, pp. 229-243, 1988.

[35] A. Nashashibi, F. Ulaby, Millimiter-Wave Polarimetric Bistatic Radar Scattering from Rough Soil Surfaces, IGARSS 2003, Toulouse, France.

[36] P. Dubois-Fernandez, H. Cantalloube, B. Vaizan, G. Krieger, R. Horn, M. Wendler, and V. Giroux, ONERA-DLR Bistatic SAR Campaign: Planning, Data Acquisition, and First Analysis of Bistatic Scattering Behavior of Natural and Urban Targets. To appear in IEE Proc. Radar, Sonar, Navigation, 2005.

[37] G. Yates, A.M. Horne, A.P. Blake, R. Middleton, D.B. Andre, Bistatic SAR Image Formation, EUSAR 2004, Ulm, Germany.

[38] F. Leberl, Radargrammetry, Chapter 4 in F. Henderson: Manual of Radar Remote Sensing, Am. Soc. for Photogrammetry and Remote Sensing, 1998.

[39] A. Farina, Tracking Function in Bistatic and Multistatic Radar Systems, IEE Proc. F, Vol. 133, No. 7, pp. 630-637, 1986.

[40] R.M. Goldstein and H.A. Zebker, Interferometric Radar Measurement of Ocean Surface Currents, Nature, Vol. 328, No. 20, pp. 707-709, 1987.

[41] R. Romeiser, M. Schwäbisch, J. Schulz-Stellenfleth, D. Thompson, R. Siegmund, A. Niedermeier, W. Alpers, S. Lehner, Study on Concepts for Radar Interferometry from Satellites for Ocean (and Land) Applications, 2002, www.ifm.uni-hamburg.de/ wwwrs/modeling/html/pro_kor.html

[42] A. Moccia, G. Rufino, Spaceborne Along-Track SAR Interferometry: Performance Analysis and Mission Scenarios, IEEE Trans. Aerospace Electronic Systems, Vol. 37, No. 1, pp. 199-213, 2001.

[43] E. Gill, H. Runge, A Tight Formation Flight for Along-Track SAR Interferometry, Proc. of Formation Flying Symposium 2004, Washington, USA.

[44] R. Carande, Dual Baseline and Frequency Along-Track Interferometry, Proc. IGARSS 1992, Houston, Texas, USA.

[45] F. Lombardini, F. Bordoni, F. Gini, L. Terrazzani, Multibaseline ATI-SAR for Robust Ocean Surface Velocity Estimation, IEEE Tran. Aerospace Electron. Systems, Vol. 40, No. 2, pp.417-433, 2004.

[46] K. Marais, R. Sedwick, Space Based GMTI Using Scanned Pattern Interferometric Radar, IEEE Aerospace Conference, 2001.

[47] N.A. Goodman and J.M. Stiles, A General Signal Processing Algorithm for MTI with Multiple Receive Apertures, IEEE Radar Conf., Atlanta, pp. 315-320, 2001

[48] J.Ender, Spacebased SAR/MTI using Multistatic Satellite Configurations, EUSAR 2002, pp. 337-340, Cologne, Germany.

[49] D. Cerutti-Maori, Performance Analysis of Multistatic Configurations for Spaceborne SAR/MTI Based on the Auxiliary Beam Approach, EUSAR 2004, pp. 631-634, Ulm, Germany.

[50] D. Hounam et al.., An Autonomous, Non-Coperative, Wide Area Traffic Monitoring System using Space-Based Radar (TRAMRAD), IGARSS 2005, Seoul, Korea.

[51] A. Gabriel, R. Goldstein, H. Zebker, Mapping Small Elevation Changes over Large Areas: Differential Radar Interferometry, J. Geophys. Res., Vol. 94, 1989.

[52] D. Massonnet, K. Feigl, Radar Interferometry and its Application to Changes in the Earth's Surface, Review of Geophysics, Vol. 36, No. 4, pp. 441-499, 1998.

[53] A. Ferretti, C. Prati, F. Rocca, Nonlinear Subsidence Rate Estimation using Permanent Scatterers in Differential SAR Interferometry. IEEE Trans. Geosci. Remote Sens. Vol. 38(5), pp. 2202-2212, 2000.

[54] F. Lombardini, Differential Tomography: A New Framework for SAR Interferometry, Vol. 43, No. 1, pp. 37-44, 2005.

[55] A. Moreira, G. Krieger, J. Mittermayer, Comparison of Several Bi-Static SAR Configurations for Spaceborne SAR Interferometry, Proc. ASAR Workshop 2001 (see also US Patent No. 6,677,844 B2, 2002).

[56] H. Fiedler, G. Krieger, Close Formation of Micro-Satellites for SAR Interferometry, Proc. of Formation Flying Symposium 2004, Washington, USA.

[57] G. Krieger, M. Rodriguez Cassola, M. Younis, R. Metzig, Impact of Oscillator Noise in Bistatic and Multistatic SAR, IGARSS 2005, Seoul, Korea.

[58] M. Eineder, Oscillator Clock Drift Compensation in Bistatic Interferometric SAR, IGARSS 2003, Toulouse, France.

[59] R. Metzig, Comments to the Synchronisation of Bistatic Interferometric SAR, Sep. 2003

[60] Evans, N.B., Lee, P., Girard, R., The RADARSAT-2/3 Topographic Mission, EUSAR 2002, pp. 37-39, Cologne, Germany.

[61] V. Candelier, P. Canzian, J. Lamboley, M. Brunet, G. Santarelli, Space Qualified 5 MHz Ultra Stable Oscillators, Intern. Frequency Control Symp. 2003, pp. 575582, Tampa, Fl., USA. 